\title{
Rotationally Gated Fluorescence: Common Theme Behind Molecular Rotors, Photoactivation and Förster Resonance Energy Transfer (FRET)
}

\author{
László Bene* and László Damjanovich \\ Department of Surgery, Faculty of Medicine, University of Debrecen, Hungary \\ *Corresponding author: László Bene, Department of Biophysics and Cell Biology, University of Debrecen, Debrecen, \\ H-4032 Egyetem tér 1; Mail: H-4002 Debrecen P.O.Box 400, Hungary
}

\section{ARTICLE INFO}

Received: 蔧 March 03, 2020

Published: March 12, 2020

Citation: L. Bene, L. Damjanovich. Rotationally Gated Fluorescence: Common Theme Behind Molecular Rotors, Photoactivation and Förster Resonance Energy Transfer (FRET). Biomed J Sci \& Tech Res 26(4)-2020. BJSTR. MS.ID.004370.

\section{ABSTRACT}

About the common features behind the mechanism of molecular rotors, photoswitching of engineered fluorescent proteins (GFPs), and Förster resonance energy transfer (FRET). Besides explining the common mechanism, we also offer a new method for increasing sensitivity of viscosity determination even in living cells, by the combination of polarization energy transfer (polFRET) method and a molecular rotor dye either as an energy donor or acceptor.

Keywords: Internal Charge Transfer (TICT) State; Viscosity; Fluorescence Lifetime; Quantum Yield; Photoactivation; Polarization Energy Transfer (polFRET)

Abbreviations: FRET: Förster Resonance Energy Transfer; FRAP: Fluorescence Recovery After Photobleaching; LE: Locally Excited State

\section{Introduction}

Viscosity represents the strength of interaction of an object or a molecule with other objects or molecules within immediate vicinity in a solution. Its importance follows from the fact that via its involvement in the lateral and rotational diffusion coefficients it determines the rates of biochemical-biological processes in almost every area in life sciences. From another point of view, it determines also the rates of energy dissipation of moving bodies, molecules. An interesting feature is that while lateral diffusion depends on the inverse 1st power of the object radius, the rotational one depends on the inverse 3 rd power of the radius, i.e. on the volume [1], suggesting a rule - which is a rather general one, having significance also in fluorescence - that by increasing dimensionality of motion, the degrees of freedom for energy dissipation is also increased, exemplified by translation and rotation as 1- and 3-D motions (Figure 1, Panel A).
Tradional approaches of viscosity measurements in cell biology rest on Fluorescence Recovery After Photobleaching (FRAP) mea surements of translational diffusion and fluorescence or phosphorescence anisotropy measurements of rotational diffusion [2-6]. In FRAP, lateral diffusion is quantified by the speed of reappearance of fluorescence in a target volume after selectively destroying by intense light a portion of fluorophores initially present there [2,3]. In fluorescence anisotropy measurements, rotational diffusion is quantified by the speed of randomization of polarization directions of the emitted photons after excitation by linearly polarized light [4-6]. These two approaches, and even their combinations in non-imaging and imaging modes, could be realized equally well in the cytoplasm, on the cell membrane, and in the different intracellular organelles and/or in their membranes [2,6]. Substantial interest has been arisen by a class of special dyes called molecular rotors [1] (Figure 1, Panel B). 
A)

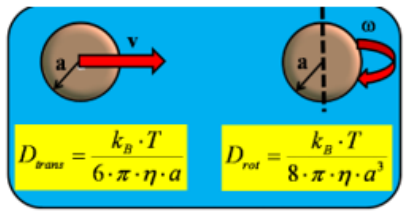

B)

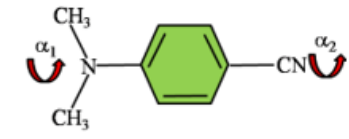

C)

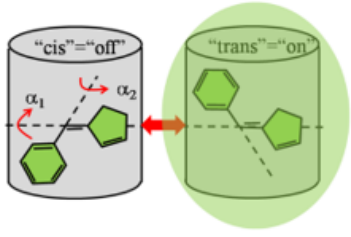

D)

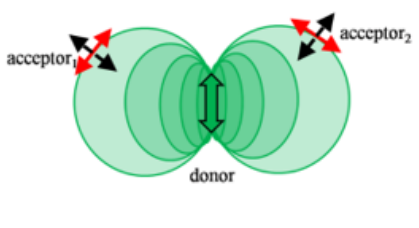

Figure 1: Rotational gate mechanisms of energy dissipation.

Panel A: Envisioned is that energy dissipation towards the bathing environment for a body making rotational diffusion is at a higher rate than for a body making translational diffusion. This follows from the fact that, while the magnitude of the driving force for both the translational and rotational diffusions is the same thermal energy, the damping force due to the immediate neighborhood of bodies - expressed by the denominators of the formulae for the diffusion constants Dtrans, Drot - is much larger for the rotating than for the translating body. Besides the difference in the constants (8 for rotation vs. 6 for translation) in the case of rotation the viscous drag is determined by the 3rd power of the radius "a" (i.e. the body volume) in contrast to the much weaker linear dependence for translation [1]. These formulae ("Deby-Stokes-Einstein") are based on that in thermal equilibrium the rate of energy uptake by thermal collisions equalizes the rate of energy loss due to the viscous drag. $\mathrm{kB}$ : Boltzmann's Constant, T: Absolute Temperature. The difference in the rates of energy dissipation can also be connected to the dimensionality of the process: while translation is 1-dimensional process, rotation is 3-dimensional one.

Panel B: Example of the rotor dye, a member of the aniline nitrile family, 1,4-dimethylamino benzonitrile (DMABN) [7,8]. Excitation with light is followed by an increase of dipole moment which in turn leads to a rotation of the $\mathrm{N}\left(\mathrm{CH}_{3}\right)_{2}$ and $\mathrm{CN}$ groups around the bonds connecting to the benzene ring, i.e. a twisted state of the original planar molecule is assumed, due to the interaction of the increased dipole with the environment. Upon this twisting a new excited state, called "twisted internal charge transfer" (TICT) state is achieved, in which the charge is separated from the electron donating (dimethylamino) end to the electron accepting (nitrile) end, and which has a lower ground-excited state energy gap than that of the initially excited one, the "locally excited" (LE) state. The increase in dimensionality due to twisting, and the increased charge separation, lead to increased environmental interactions manifested in reduced ground-excited state energy gap, or what is equivalent, in redshifted fluorescence, and reduced fluorescence lifetime and quantum efficiency. At the limiting case, de-excitation from the TICT state - for too small ground-excited state energy gaps - can happen totally non-radiatively. The rate of twisting transition from the LE to the TICT state might depend on viscosity, polarity, and $\mathrm{pH}$ of the environment. Sensitivity on these factors can be optimised by engineering the proper dye-structure. Concerning viscosity, generally valid is that by increasing viscosity, lifetime, quantum efficiency and intensity increase, and the color of fluorescence might be blue-shifted. Although rotors can be high-tuned to be sensitive only to viscosity, even in this case their response can be highly diverse. This is because of the complexity of viscosity itself: E.g. diffusion can be of a mixture of the slipping and hopping mechanisms, for the high and low solute-per-solvent size-ratios, respectively [1].

Panel C: Depicted is a common mechanism of photo-activation or photo-switching of some genetically engineered visible fluorescing proteins (VFPs). The decisive step is again a rotation, but of 2 chromophore rings relative to each other, the "cistrans isomerisation" [10]. While the "cis" state does not support extended electron resonance capable for fluorescence, because the 2 choromophore rings are not in the same plane, the "trans" state does it. The "on" state can be achieved by illumination with short-wavelength light in the blue, near-UV regions. In contrast, the "off" state can happen by alone after some cycles of excitation-deexcitation events, or can be aided also with light. The "cis-trans isomerisation" transition can depend on the protonation state of the choromophores. The protonation-deprotonation equilibrium is the factor which can be shifted by the relatively high energy switching light beams [11].

Panel D: Concerning principle of operation, a donor-acceptor FRET-pair constitutes a molecular rotor. Assuming favorable spectral overlap and short donor-acceptor separation, an acceptor can only take over energy from the donor if the absorption moment of acceptor is close to parallel with the local field of donor (red double-arrows) at the position of acceptor. There is no energy transfer for perpendicular local field-dipole orientations (black double-arrows), regardless of the shortage of distance. The functional dependence of the acceptor position and the local field-dipole relative orientation is described by the orientation factor for FRET $\left(\mathrm{k}^{2}\right)$. Depending on the direction of acceptor position and the relative local field-dipole orientation $\mathrm{k} 2 \mathrm{varies}$ between zero and 4 . When these 2 directions rapidly randomize before FRET takes place - termed dynamic-isotropic random averaging condition -, the orientational average value of $k^{2}$ is $2 / 3[12,13]$. It can now be conveniently imagined that for an acceptor dipole making random Brownian rotations, $\mathrm{k}^{2}$ might increase due to the more frequent orietational matching, i.e. $\mathrm{k} 2$ acts as an orientational switch or gate [14-16]. As consequences, just as for the rotor dyes, quantum yield and lifetime of donor diminish, and the emission by the FRET pair suffers a red-shift in color. As it can be seen, the FRET-pair inherently conveys the basic properties of a rotor dye, however, it has a larger inertia, i.e. the spatial and time resolution of the response of the FRETpair is behind the rotor-dye. While with the FRET pair 2 distinct dyes carries out the sensing, requiring $\sim$ nsec response time, in contrast with the rotor dyes 2 segments within a single dye, requiring only a $\sim$ psec response time. 
These are small compounds having several aromatic rings with substantial rotational freedoms around one or more bonds. They have two excited states: In the Locally Excited One (LE) all their rings are in a single plane leading to high quantum efficiency because of the suppression of rotations. In the other, called "Twisted Internal Charge Transfer" (TICT) state, the molecule assumes a 3-D state instead of the original 2-D one, with one or more rings being rotated out from the LE plane [1,7]. Because the dipole moment of the dye is also increased here, this state has also a higher reactivity with the neighborhood, implying also a more efficient loss of energy and a lower quantum efficiency. The point in this process is that the transition from the 2-D planar state to the 3-D non-planar one is basically dictated by the local viscosity and polarity in the immediate vicinity of the dye, with relative sensitivities to these factors controlled by chemical engineering of side groups. As an extreme case, molecular rotors sensitive to only viscosity can be achieved.

This process can also be envisioned as a special type photo-switching between the high quantum efficiency planar state and the lower quantum efficiency TICT state (Figure 1, Panel C). The transition to the TICT state may also be followed by a red-shift in the color of emission for TICT state energy gaps slightly lower than in the LE state. Furthermore, fluorescence emission might also completely cease for much lower TICT state energy gaps. Experimentally, molecular rotors can quantify an increasing local viscosity by increasing fluorescence lifetime, quantum efficiency, fluorescence intensity, and a blue-shifted fluorescence color $[7,8]$. The outstanding feature of molecular rotors distinguishing them from polarization-based sensing of viscosity, is the much higher sensitivity, due to the fact that only the rotation of a subportion of a dye occurring in $\sim$ psec time is involved, i.e. due to the much smaller inertia [7]. Rotor dyes can be engineered not only for sensing viscosity, but also for indicating contact points between objects, and probing binding reactions [9].

In their work, H Ying et al. [10] developed a rotor dye by a series of chemical modification of BODIPY for serving two purposes at the same time: The 1st purpose is to enable it for adhering to the vicinal dithiol moieties of endoplasmatic reticulum nascent proteins - via an introduction of an arsenicate moiety - and triggering autophagy of endoplamic reticulum ("reticulophagy") by this binding. The 2nd purpose is the quantitation of this binding via the above "rotor effects". The authors have chosen fluorescence lifetime as the probe parameter, to exclude possible disturbances due to changes in local dye concentrations inherent in intensity measurements. An advantage of the rotor dye is that it is adequate to apply a single emission color channel for quantitating viscosity. The other detection channels might be preserved for other dyes for the localization of rotor dye accumulation e.g. lysotracker, mitotracker for lysosomes and mitochondria, respectively. In the above we saw that excitation induced segmental rotation of certain dyes damped by local viscosity may act as a kind of a photoswitching process.
Actually, the operation of the "official" photoswitching or photoactivation phenomenon taking place in certain engineered Visible Fluorescent Proteins (VFPs) rests on a similar principle [11,12]. There the chomophore suspended in a b-barrel shield might have large enough rotational freedom to sample different orientations after excitation. The photo-activation property is conveyed by the circumstance that these sampled orientations might have different quantum yields due to the different degree of quenching by the amino acid side chains of the b-barrel wall. The energy for the rotation of the choromophore is supplied by the excitation: The increased dipole moment of the chromophore upon excitation results in increased torque exerted by the neighboring amino acid side chains of the b-barrel wall. Photoactivation might be tuned by the careful engineering of chromophore as well as the quencher amino acid side chains down the barrel wall. The conventional Förster Resonance Energy Transfer (FRET) pair has already the main characteristics of a rotor dye, notwithstanding now that the relative orientation of two different dyes is that what is important (Figure 1, Panel D).

The conventional Förster responsible for switching off the donor - and switching on the acceptor - at a given donor-acceptor distance is the relative orientation of the absorption dipole of acceptor and the donor local field at the position of acceptor $[13,14]$. The functional form of this dependence is conveyed by orientation factor for FRET $\left(\mathrm{k}^{2}\right)$. At each position around the donor it is generally valid that $\mathrm{k}^{2}$ is maximal when the acceptor dipole is parallel, and zero when it is perpendicular with the direction of donor local field at that special position. It also can be suspected that by increasing the speed of rotation of either of the donor or acceptor might lead to increased FRET, by the increased frequency of parallel encounters $[15,16]$ (Figure 2). To put it another way, $\mathrm{k}^{2}$ and FRET efficiency might be increased by reducing local viscosity $[15,17]$. FRET has already been combined with the rotor dyes for sensing local viscosity $[7,18]$. The problem of sensing viscosity via rotor intensity is that intensity depends not only on viscosity but also on the concentration of the rotor dye.

In the "ratiometric" arrangement, a rotor dye and a conventional one are coupled together with a flexible linker realizing a FRET-pair of the 1-to-1 donor-acceptor ratio. Here the fluorescence emission of the conventional dye - supposed to be insensitive to viscosity - is used for probing local concentration of FRET-pair and rotor dye, and the fluorescence of the rotor for quantitating viscosity. Recently "polarization energy transfer" (polFRET) methods has been introduced to monitor donor and acceptor dye rotations at different distances [19,20] (Figure 3). Here, polarized donor and acceptor intensity components are measured simultaneously, enabling quantitation of donor and acceptor anisotropies beside FRET efficiency. Although this method as it stands might already be useful for sensing viscosity, its capabilities might be improved by the involvement of rotor dyes: 


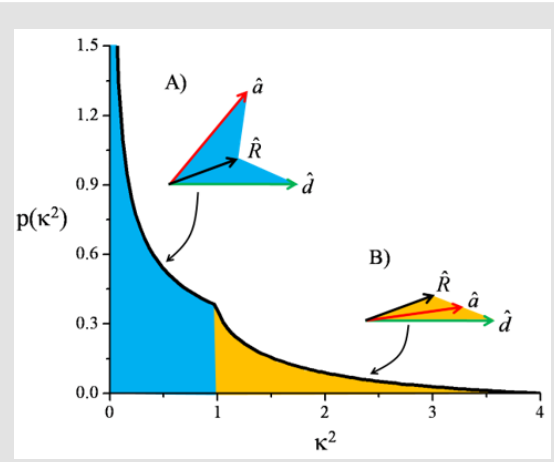

Figure 2: Effect of spatial dimensionality on FRET. Probability density function of orientation factor for FRET $\left(k^{2}\right)$, with insets A) and B) depicting unit vectors showing the relative orientations of the donor emission dipole (d), acceptor absorption dipole (a) and acceptor position relative to the donor position (R). Inset A): In the majority of cases the three unit vectors are not in the same plane, but they sample three mutually different spatial directions. In these cases the orientation factor is relatively low $\left(0<\mathrm{k}^{2}<1\right.$, blue shaded area under the $\mathrm{p}\left(\mathrm{k}^{2}\right)$ curve), corresponding to a relatively weak FRET, even for small donor-acceptor distances. Inset B): In a fewer number of cases, the three unit vectors are in the same plane (coplanar). In these cases the orientation factor is higher $\left(1<\mathrm{k}^{2}<4\right.$, orange shaded area under the $\mathrm{p}\left(\mathrm{k}^{2}\right)$ curve), corresponding to higher FRET, even for large donor-acceptor distances. Increasing rotational speed either for the donor of the acceptor the frequency of coplanar encounters of the three vectors also increases leading to a more effective loss of donor's excitation energy via FRET ("rotational gate" mechanism).

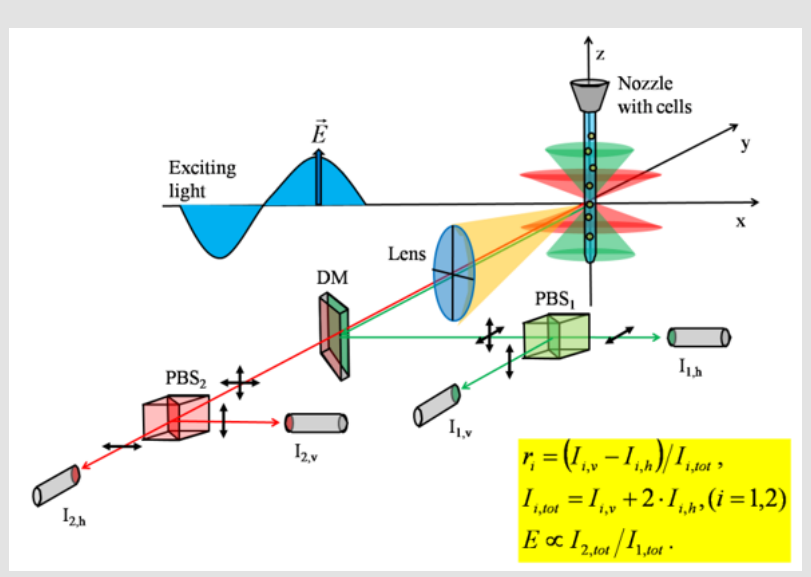

Figure 3: "Single-laser polarization FRET (polFRET)" scheme [19, 20]. Rotor dyes can be used as a donor or acceptor of a FRETpair, in the polFRET measuring scheme, either for a ratiometric calibration of the rotor dye, or for improving sensitivity of the polFRET method for the detection of mobility (viscosity) changes. Vertically polarized light traveling in the x-direction excites the donor-acceptor FRET-pairs on the cell surface at the origin of the xyz coordinate frame. The green and red double-cones coinciding with their apexes indicate the orientational distributions of the donor and acceptor emission dipoles. If we take the orientational distributions for the donor and acceptor the same in the absence of FRET, then the orientational distribution of donor dipoles is always narrower than for the acceptor dipoles, because the hyperpolarizing and depolarizing effects of FRET, respectively. On the donor side there is shorter duration of time for physical rotation and homo-FRET to take place, because of the reduction of lifetime by FRET. On the acceptor side, the acceptor anisotropy is the intensity-weighted average on the directly excited and sensitized emission anisotropies, with the latter one being practically zero. The collecting lens collects all fluorescence in the orange sterical cone from the cell momentarily positioned at its focus. DM dicroic mirror separates the donor and acceptor fluorescences (green, red) and projects them into the polarizing beam splitter cubes PBS1, PBS2, which dissect the beams into the vertically and horizontally polarized components, designated also by vertical and horizontal black double-arrows. The polarized intensity components are detected by photomultipliers (grey cylinders). Scattered exciting light is blocked and the fluorescence channels are further specified by placing band-path filters in front of the detectors (not indicated). From the polarized intensity components first, the total intensities $\left(\mathrm{I}_{1, \text { tot }}, \mathrm{I}_{2, \mathrm{tot}}\right)$, then the fluorescence anisotropies (r1, r2) are determined for the donor and acceptor as indicated in the yellow box. Then FRET efficiency E is computed from the total intensities $\left(\mathrm{I}_{1, \text { tot' }} \mathrm{I}_{2 \text { tot }}\right)$. Finally, the method enables correlating donor and anisotropies with the FRET efficiency, i.e. correlating donor and acceptor rotational speeds at different separation distances. 
(i) Rotor dye response might be calibrated in the above-described "ratiometric" fashion, when the intensity of conventional dye is used for concentration indicator. A direct measurement of also the rotational correlation time of the conventional dye, could serve as another check of viscosity in addition to the rotor intensity.

(ii) Sensitivity on conformational changes and binding reactions of the polFRET method might be improved with rotor dyes. If e.g. rotor is applied as donor and conventional dye as acceptor, increase in viscosity would introduce increase in donor and acceptor anisotropies, and quantum efficiency of the donor. Even if donor-acceptor distance is left unaltered, and $\mathrm{k}^{2}$ might diminish with increasing viscosity, an increase in FRET efficiency could still be anticipated based on the dependence of FRET rate constant on the donor quantum yield.

If, however, by reversing the above scheme, rotor would be applied as acceptor and conventional dye as donor, donor and acceptor anisotropies and acceptor emission intensity would increase with viscosity. At a fixed donor-acceptor separation, FRET efficiency would now only decrease due to the decrease of $\mathrm{k}^{2}$ with increasing viscosity. Donor and acceptor anisotropy enhancements would serve as a check for the intensity enhancement of the rotor with increasing viscosity, valid also for the previous case.

\section{Conclusion}

We revealed parallel features behind the working meahanisms of molecular rotors, photoswitching of GFPs, and the FRET process. Polarisation FRET (polFRET) method is suggested in combination with a molecular rotor either as a donor or an acceptor for increasing the sensitivity of viscosity measurements in a fluorescent microscope or in a flow cytometer.

\section{Acknowledgements}

Financial support to L.B. for this work was provided by TÁMOP4.2.2.A-11/1/KONV-2012-0045 project co-financed by the European Union and the European Social Fund, OTKA Bridging Fund support OSTRAT/810/213 by the University of Debrecen. L.B. is wishing to express the appreciation for Imre Péntek (Nagykőrös, Hungary) for a fascinating first introduction into the field of wave polarization.

\section{References}

1. Valeur B (2002) Microviscosity, fluidity, molecular mobility. Estimation by means of fluorescent probes (Chapter 8). In: Molecular fluorescence: Principles and applications. Wiley-VCH, Weinheim, 226-246.

2. Deschout H, Hagman J, Fransson S, Jonasson J, Rudemo M, et al. (2010) Straightforward FRAP for quantitative diffusion measurements with a laser scanning microscope. Opt Exp 18(22): 22886-22905.
3. Swaminathan R, Bicknese S, Periasamy N, Verkman AS (1996) Cytoplasmic viscosity near the cell plasma membrane: Translational diffusion of a small fluorescent solute measured by total internal reflection-fluorescence photobleaching recovery. Biophys J 71(2): 11401151.

4. Valeur B (2002) Fluorescence polarization. Emission anisotropy. Chapter 5 In: Molecular fluorescence. Principles and applications. WileyVCH Weinheim, 125-154.

5. Austin RH, Chan SS, Jovin TM (1979) Rotational diffusion of cell surface components by time-resolved phosphorescence anisotropy. Proc. Natl. Acad. Sci. USA, 76(11): 5650-5654.

6. Velez M, Axelrod D (1988) Polarized fluorescence photobleaching recovery for measuring rotational diffusion in solutions and membranes. Biophys J 53(4): 575-591.

7. Haidekker MA, Theodorakis EA (2010) Environment-sensitive behavior of fluorescent molecular rotors. J Biol Eng 4(11): 1-14.

8. Kuimova MK (2012) Molecular rotors image intracellular viscosity. Chimia 66(4): 159-165.

9. Suhina T, Weber B, Carpentier CE, Lorincz K, Schall P, et al. (2015) Fluorescence microscopy visualization of contacts between objects. Angew Chem Int Ed 54(12): 3688-3691.

10. He Y, Shin J, Gong W, Das P, Qu J, et al. (2019) Dual- functional fluorescent molecular rotor for endoplasmic reticulum microviscosity imaging during reticulophagy. Chem Commun 55(17): 2453-2456.

11. Patterson GH (2014) Optical highlighter photophysical properties. Ch. 6 in The fluorescent protein revolution. RN Day, MW Davidson (Eds.), Series in cellular and clinical imaging, (edn.). A Periasami, CRC Press, Taylor\&Francis Grou, pp. 123-156.

12. Schäfer LV, Groenhof G, Boggio Pasqua M, Robb MA, Grubmüller H (2008) Chromophore protonation state controls photoswitching of the fluoroprotein as FP595. PLOS Comp Biol 4(3): e1000034.

13.van der Meer BW (2013) Optimizing the orientation factor KAPPASQUARED for more accurate FRET measurements (Chapter 4) In FRET - Förster resonance energy transfer: from theory to applications. In: I Medinzt, N Hildebrandt (Eds.) Wiley-VCH; (1 ${ }^{\text {st }}$ edn.): 63-104.

14. van der Meer BW (2002) Kappa-squared: from nuisance to new sense. Rev Mol Biotechnol 82(3):181-196.

15. Xu QH, Wang S, Korystov D, Mikhailovsky A, Bazan GC, et al. (2005) The fluorescence resonance energy transfer (FRET) gate: A time-resolved study. Proc Natl Acad Sci USA 102(3): 530-535.

16. Fábián ÁI, Rente T, Szöllősi J, Mátyus L, Jenei A (2010) Strength in numbers: Effects of acceptor abundance on FRET efficiency. Chem Phys Chem 11(17): 3713-3721.

17. Somogyi B, Norman JA, Rosenberg A (1986) Gated quenching of intrinsic fluorescence and phosphorescence of globular proteins. An extended model. Biophys J 50(1): 55-61.

18. Haidekker MA, Brady TP, Lichlyter D, Theodorakis EA (2006) A ratiometric fluorescent viscosity sensor. J Am Chem Soc 128(2): 398399.

19. Bene L, Gralle M, Damjanovich L (2018) Confocal microscopic dual-laser dual-polarization FRET (2polFRET) at the acceptor side for correlating rotations at different distances on the cell surface. BBA Gen 1862(4): 1050-1068.

20. Bene L, Bagdány M, Ungvári T, Damjanovich L (2018) Dual-laser tetrapolarization FRET (4polFRET) for site-selective control of homo-FRET in hetero-FRET systems on the cell surface: the homo-FRET gate. Anal Chem 90(17): 10159-10170. 
ISSN: 2574-1241

DOI: $10.26717 /$ BJSTR.2020.26.004370

László Bene. Biomed J Sci \& Tech Res

(c) (i) This work is licensed under Creative

Submission Link: https://biomedres.us/submit-manuscript.php

$\begin{array}{ll}\text { BIOMEDICAL } & \text { Assets of Publishing with us } \\ \text { RESEARCHES } & \text { - Global archiving of articles } \\ \text { - Immediate, unrestricted online access } \\ \text { - Rigorous Peer Review Process } \\ \text { https://biomedres.us/ }\end{array}$

